\title{
Rectal arteriovenous malformation: an unusual emergency
}

\author{
Sourav Choudhury ${ }^{1}$, Sasi Mouli ${ }^{2}$ \\ ${ }^{1}$ Sanjay Gandhi post graduate Institute of Medical sciences, India \\ ${ }^{2}$ Kings George Medical College, India
}

Key words: Arteriovenous malformation; rectum; per rectal bleed; CT angiography; low anterior resection

\section{Introduction}

We herein report a case of rectal arteriovenous malformation (AVM) presented to us with massive bleeding per rectum with haemorrhagic shock. He was admitted and resuscitated with intravenous fluid and blood transfusion. Colonoscopic control of bleeding was unsuccessful and the patient was successfully treated with laparoscopic low anterior resection and diversion ileostoma. He did not have rebleeding after 6 months follow up. Rectal AVM is rare and bleeding rectal AVM requiring emergency is very rare. So this case has been reported.

\section{Presentation}

A 52 year male patient presented with bleeding per rectum for 3 months. Bleeding was painless, intermittent and large in quantity requiring multiple blood transfusions. He had a history of haemorrhoidectomy 3 years back. In the emergency room, he had tachycardia, pallor and hypotension. He doesn't have any history of anticoagulant therapy. Abdominal examination was unremarkable. Per rectal examination did not reveal any mass or haemorrhoids. Urgent colonoscopy was planned. It revealed dilated submucosal vessels in rectum with multiple active bleeding points. Rest of the large bowel was normal. He was admitted and resuscitated with intravenous fluid and blood transfusion. Blood investigations revealed Haemoglobin $7.8 \mathrm{~g} / \mathrm{dL}$, Platelet 200 000/dL, Creatinine $0.9 \mathrm{mg} / \mathrm{dl}$. CT angiography abdomen revealed multiple dilated vascular lesion in the mesorectum (Figure 1).

No mass lesion found in CT scan. Laparoscopic Low anterior resection was done with a diversion loop ileostomy. Gross specimen revealed dilated vascular lesion in the rectum (Figure 2). Intraoperatively no vascular anomaly found in the pelvis. Histopathology confirms arteriovenous malformation (AVM) of the rectum. Postoperatively he recovered well and after 6 months of surgery, he doesn't have any bleeding episode.

Correspondence: Sourav Choudhury

E-mail: drcsourav@gmail.com

Received: 11-03-2019 Accepted: 18-07-2019

(iD http://orcid.org/0000-0002-3012-6988

DOI: http://doi.org/10.4038/sljs.v37i2.8628

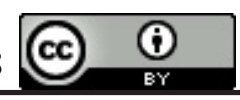

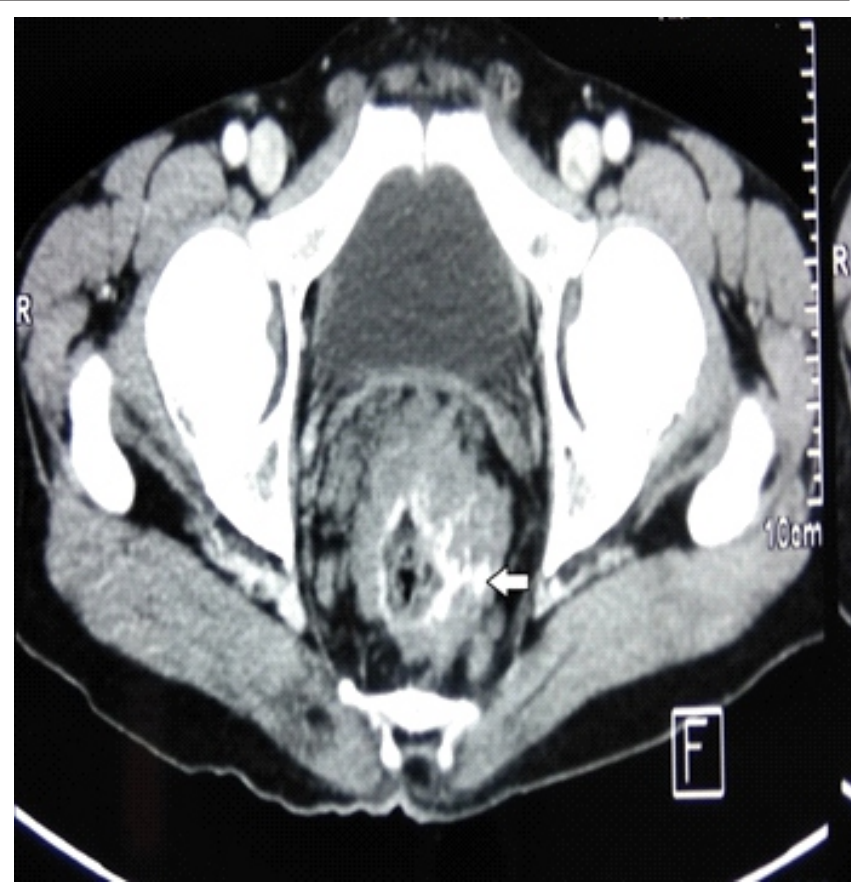

Figure 1. Axial cut in CT scan showing active blushing of contrast

\section{Discussion}

Arteriovenous malformation (AVM) in the GI tract is also known as vascular ectasia or angiodysplasia. It is commonly seen in children, but cases in adults are also being reported. Incidence of AVM is reported 1.4-3\% in cases of lower GI bleeding - out of this figure, only $0.9 \%$ AVM has been reported in the rectum. This figure is quite high $-8 \%$ according to Japanese literature [1]. No data are available regarding the incidence in India. Most common location of AVM is caecum (74\%), other sites are rectum, jejunum, duodenum and stomach according to decreasing order of frequency. In the paediatric population, the incidence of rectal $\mathrm{AVM}$ is as high as $14 \%$.

Considering the high incidence in the paediatric population, aetiology of rectal AVM is supposed to be congenital. Japanese literature showed many cases of rectal AVM are associated with hypertension, cardiovascular disease and pulmonary fibrosis, suggesting vascular degeneration as a probable aetiology in the development of AVM. They also reported that many patients had a history of haemorrhoidectomy in the past, like in our case. Goenka et al. Showed 


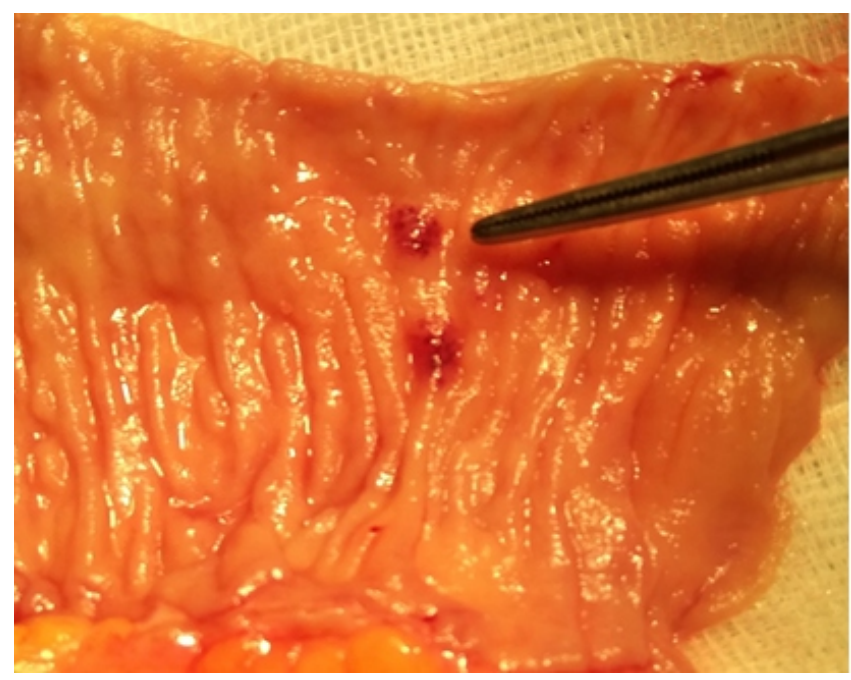

Figure 2. Surgical specimen with AVM pointed by tip of forceps

$89 \%$ of patients of cirrhosis with portal hypertension have recto sigmoid AVM [2].

A most common presentation of rectal AVM is bleeding per rectum, although a significant number of patients are asymptomatic and diagnosed incidentally. Often patient gives a history of haemorrhoidectomy but considering patient age and other symptoms, a colonoscopy may be warranted to diagnose rectal AVM. Moore et al. Proposed a classification of GI AVM based on their experience: Type 1 AVM is solitary, small lesion not palpable from the serosal side, usually in the right colon and occurs in an elderly patient with age more than 50 years. Type 2 AVM are larger, congenital and usually occurs in children and young patients. Type 3 AVM is associated with hereditary haemorrhagic telangiectasia.[3] This classification does not include rectal AVM which is common in children(14\%) and cirrhotic patients and we proposed Type 4 AVM which includes the rectosigmoid AVM.

Recommended treatment options for rectal AVM are surgical resection, laser photocoagulation [4], transarterial embolization and Balloon-occluded retrograde transvenous embolization [5]. Surgical treatment is preferred in hemodynamically unstable patients and if other facilities are not available. Available surgical treatment options are Low anterior resection, trans-sacral resection, transanal resection and transanal suture ligation. Nishimuta $\mathrm{Y}$ et al. Demonstrated the efficacy of laser photocoagulation in the treatment of GI AVM in 59 patients, he showed $82 \%$ success rate in controlling bleeding. Rate of rebleeding is higher in multiple lesions and lesions associated with Osler Weber Rendu syndrome and von Willebrand disease. Modalities of treatment should be judged based on the available expertise and clinical condition of the patient.

\section{Conclusion}

Gastrointestinal AVM is a rare disease which often causes significant bleeding and hemodynamic instability. Various surgical and nonsurgical treatment options are available. Depending on the patient condition and availability of expertise, treatment should be individualised. Proper reporting and documentation needed for determining the prevalence of rectal AVM.

All authors disclose no conflict of interest. The study was conducted in accordance with the ethical standards of the relevant institutional or national ethics committee and the Helsinki Declaration of 1975, as revised in 2000 .

\section{References}

1. Komekami Y, Konishi F, Makita K, Mijin T, Onogawa A, Chochi $\mathrm{T}$, et al. Rectal arterio-venous malformation (AVM) with bleeding of an internal hemorrhoid. Clinical Journal of Gastroenterology [Internet]. Springer Nature; 2016 Feb;9(1):22-6. http://doi:10.1007/s12328-016-0629-8

2. Goenka MK, Kochhar R, Nagi B, et al. Rectosigmoid varices and other mucosal changes in patients with portal hypertension. Am J Gastroenterol. 1991;86(9):1185-9. PMID:1882798

3. Moore JD, Thompson NW, Appelman HD, Foley D. Arteriovenous Malformations of the Gastrointestinal Tract. Arch Surg. 1976;111(4):381-389.

http://doi:10.1001/archsurg.1976.01360220077013

4. Rutgeerts P, Van Gompel F, Geboes K, Vantrappen G, Broeckaert L, Coremans G. Long term results of treatment of vascular malformations of the gastrointestinal tract by neodymium Yag laser photocoagulation. Gut. 1985;26(6):586-93. PMID:3874122

5. Mitsuzaki K1, Yamashita Y, Utsunomiya D, Sumi S, Ogata I, Takahashi M, Kawakami S, Ueda S. Balloon-occluded retrograde transvenous embolization of a pelvic arteriovenous malformation. Cardiovasc Intervent Radiol. 1999 NovDec;22(6):518-20. PMID:10556414

\section{Learning Points:}

- Per rectal bleeding may be massive and the cause may often be very unusual

- Surgical management is the last resort if bleeding is not controlled by endoscopy or radiological interventions 